\title{
Association of Health Status and Smoking Behaviors with SARS-CoV-2 Positivity Rates
}

Thomas Duszynski ( $\nabla$ tduszyns@iu.edu )

Indiana University Richard M. Fairbanks School of Public Health at IUPUI

William Fadel

Indiana University Richard M. Fairbanks School of Public Health at IUPUI

Kara Wools-Kaloustian

Indiana University School of Medicine

\section{Brian Dixon}

Regenstrief Institute

\section{Constantin Yiannoutsos}

Indiana University Richard M. Fairbanks School of Public Health at IUPUI

\section{Paul Halverson}

Indiana University Richard M. Fairbanks School of Public Health at IUPUI

Nir Menachemi

Indiana University Richard M. Fairbanks School of Public Health at IUPUI

\section{Research Article}

Keywords: Smoking, Health Status, COVID-19, SARS-CoV-2

Posted Date: December 29th, 2020

DOI: https://doi.org/10.21203/rs.3.rs-130004/v1

License: (c) (1) This work is licensed under a Creative Commons Attribution 4.0 International License.

Read Full License 


\section{Abstract}

Background Much of what is known about COVID-19 risk factors comes from patients with serious symptoms who test positive. While risk factors for hospitalization or death include chronic conditions and smoking; less is known about how health status or tobacco use is associated with risk of SARS-CoV2 infection among individuals who do not present clinically.

Methods Two community-based population samples (including individuals randomly and nonrandomly selected for statewide testing, $n=8,214$ ) underwent SARS-CoV-2 testing in nonclinical settings. Each participant was tested for current (viral PCR) and past (antibody) infection in April or June of 2020. Before testing, participants provided demographic information and self-reported health status and tobacco behaviors (smoking, chewing, vaping/e-cigarettes). Using descriptive statistics and a bivariate logistic regression model, we examined the association between health status and use of tobacco with SARS-CoV-2 positivity on either PCR or antibody tests.

Results Compared to people with self-identified "excellent" or very good health status, those reporting "good" or "fair" health status had a higher risk of past or current infections. Positive smoking status was inversely associated with SARS-CoV-2 infection. Chewing tobacco was associated with infection and the use of vaping/e-cigarettes was not associated with infection.

Conclusions In a statewide, community-based population drawn for seroprevalence studies, we find that overall health status is associated with infection rates. Unlike in studies of COVID-19 patients, smoking status was inversely associated with SARS-CoV-2 positivity. More research is needed to further understand the nature of this relationship.

\section{Background}

Early data from China identified smokers as overrepresented among severely ill COVID-19 patients (1). Comorbid conditions (which are also more common for smokers), and increased age were first identified as risk factors for severe disease and death in China $(2,3,4)$. Data from other countries also showed an increased risk of death with each additional decade of life $(5,6)$. Poor health status and the presence of immunocompromising conditions each emerged as predictive indicators of severe or fatal outcomes ( 7 , 8). Among the more than 7,000 cases of COVID-19 initially reported in the U.S., more than $37 \%$ had at least one underlying health condition that would be considered a risk factor for severe disease, hospitalization, and intensive care with mechanical ventilation (8). Importantly, most of the existing epidemiological information was based on those with severe disease because asymptomatic and mildly symptomatic individuals are not routinely tested in the United States nor many other countries.

Thus, it is unknown how specific health behaviors and overall health status are associated with SARSCoV-2 infection, including mild infection, as opposed to moderate-to-severe cases, which have resulted in hospitalization or death. Smoking, vaping, and other uses of tobacco products are significant risk factors for multiple poor health outcomes in a population $(9,10)$. Both smoking and vaping can weaken lung 
tissue and be a source of inflammation potentially creating an opportunity for infection to occur (11). However, while it is known that tobacco use is associated with severe disease, it is less clear if such behaviors are associated with an increased risk of infection (12).

This study is based on a large statewide community sample (including random and nonrandomly selected individuals) where the state health department tested Indiana residents for both active viral infection and the presence of antibodies for SARS-CoV-2 (13). Specifically, in this study we examine how overall health status, as well as smoking and tobacco behaviors, are associated with current and previous infection with SARS-CoV-2. Indiana has higher adult and youth smoking rates than the US; and the use of electronic cigarettes and vaping products nearly doubled in the past 6 years $(11,14)$. As such, our data represents an opportunity to understand the epidemiology of key risk factors especially among asymptomatic and mildly symptomatic individuals not included in previous research. Understanding who is most at risk for infection is paramount in the ability to control the spread and protect those at highest risk until a vaccine is widely available in the population.

\section{Methods}

In this study, we pool data from two statewide testing efforts where participants were tested for active viral infection and the presence of antibodies for SARS-CoV-2 in Indiana. The pooled data represented two separate waves that were part of a statewide prevalence study conducted April 25-29 and June 3-7, 2020 which used identical sampling strategies and methods. In each wave, some participants were randomly selected based on Indiana records from tax year 2019 and 2018 to create a de-duplicated list of Indiana residents that included those who filed or co-filed taxes and all of their dependents. Updated contact information was then merged from the Indiana Bureau of Motor Vehicles and other state databases. In an effort to address under-representation of minorities in statewide testing efforts, each wave also included outreach and open testing to a nonrandom group of racial and ethnic minorities. In each wave, the random sample was stratified by each of the state's 10 public health preparedness districts representing children and adults aged 12 years or older. In all testing, excluded were institutionalized individuals, those with an out-of-state address, and young children.

Randomly selected participants were contacted through postcards, text messages, email, and telephone and given information to either register online or speak to a live operator (15). Nonrandom outreach was done in conjunction with civic, religious, and community leaders in both the African American and Hispanic communities, to identify neighborhood centers where underserved populations could be tested. All individuals in both the random and nonrandom groups of both waves are analyzed herein to examine how health status and behaviors are associated with SARS-CoV-2 infection. This pooled group represents community-based individuals who were tested regardless of symptoms, medical indications, risk factors, and prior testing history-and is thus different than all other hospital-based groups previously studied.

Prior to testing, participants were asked to complete a form which included questions about demographic characteristics, and self-reported health status, and tobacco behaviors, which include cigarette, chewing 
tobacco or vaping usage stratified by every day, somedays, or not at all, consistent with the Behavioral Risk Factor Surveillance System (BRFSS) questionnaire (15).

Trained personnel used Dacron swabs to collect nasopharyngeal specimens for RT-PCR testing for SARSCoV-2. RT-PCR testing was conducted using the Lilly Clinical Diagnostics Lab SARS-CoV-2 test based on the CDC primary set, the Luminexon NxTAG CoV Extended Panel, or the Roche cobas SARS-CoV-2 test depending on platform availability. A venipuncture was used to obtain 2-3 $\mathrm{mL}$ of blood for antibody testing using a chemiluminescent microparticle immunoassay for detection of SARS-CoV-2 IgG (16).

\section{Statistical Analysis}

All analyses were performed using R (version 3.6.3) on the combined samples from wave 1 and 2 of the prevalence study. Descriptive statistics were calculated for the sample population using frequencies and percentages. A bivariate logistic regression model controlling for age, sex, race, ethnicity, and whether a person was randomly selected or in a supplemental sample to examine the association between both health status and use of tobacco with SARS-CoV-2 positivity on either PCR or antibody tests. The effects of sampling group and household clustering were controlled for using random effects in the model.

The Institutional Review Board (IRB) at Indiana University deemed the study as exempt from human subjects research under the public health surveillance exemption.

\section{Results}

Including both random and nonrandom samples, from two distinct waves of testing, the total sample size for this study was 8,214 individuals whose demographic characteristics are displayed in Table 1. Among five possible categories, $16 \%$ self-reported being in "excellent" health while "very good" and "good" were reported by $38 \%$ and $35 \%$, respectively. 
Table 1

Demographic characteristics of the sample including self-reported health status and tobacco use $(n=8,214)$.

\begin{tabular}{|ll|}
\hline Characteristics & Participants (\%) \\
\hline Female & $4,565(55.6)$ \\
\hline Male & $3,647(44.4)$ \\
\hline Nonite & $6,599(80.3)$ \\
\hline Hispanic & $1,615(19.7)$ \\
\hline Non-Hispanic & $653(7.9)$ \\
\hline Age: $<40$ & $7,561(92.0)$ \\
\hline Age: $40-59$ & $2,379(28.9)$ \\
\hline Age: $60+$ & $3,036(36.9)$ \\
\hline Randomly selected group & $2,799(34.0)$ \\
\hline Wave 1 & $3,658(44.5)$ \\
\hline Wave 2 & $2,668(32.5)$ \\
\hline Nonrandomly selected group & \\
\hline Wave 1 & $937(11.4)$ \\
\hline Wave 2 & $951(11.6)$ \\
\hline Self -reported Health Status & \\
\hline Excellent & $1,331(16.2)$ \\
\hline Very Good & $3,140(38.2)$ \\
\hline Good & $2,894(35.2)$ \\
\hline Fair & $699(8.5)$ \\
\hline Poor & $92(1.1)$ \\
\hline Cigarette Use & \\
\hline Everyday & \\
\hline Some days & \\
\hline Not at all & \\
\hline Current chewing tobacco use & \\
\hline
\end{tabular}




\begin{tabular}{|ll|}
\hline Characteristics & Participants (\%) \\
\hline Everyday & $115(1.4)$ \\
\hline Some days & $79(0.9)$ \\
\hline Not at all & $7,962(96.9)$ \\
\hline Current vaping or e-cigarette use & \\
\hline Everyday & $110(1.3)$ \\
\hline Some days & $126(1.5)$ \\
\hline Not at all & $7,920(96.4)$ \\
\hline
\end{tabular}

There were more than 1,300 persons who identified as using tobacco either every day or on some days. Almost $11 \%$ of respondents reported current cigarette use including $656(7.9 \%)$ who smoke every day and 224 (2.7\%) who smoked on some days. Reported use of chewing tobacco was less frequent with 115 $(1.4 \%)$ reporting using chewing tobacco daily, while $79(0.9 \%)$ chewed on some days. Vaping or ecigarettes use was reported by $2.8 \%$ including 110 individuals who reported vaping daily (1.3\%) and 126 individuals who reported vaping on some days (1.5\%).

The bivariate relationship between tobacco use and SARS-CoV-2 positivity rates (PCR and antibody) are displayed in Table 2. Increased frequency of cigarette use was inversely associated with current infection, previous infection, and overall population prevalence. For example, whereas the population prevalence among respondent who do not currently smoke cigarettes was $6.6 \%$, the population prevalence among those that report smoking everyday was $2.0 \%(p<0.001)$. 
Table 2

Bivariate Infection rates and prevalence by tobacco use

\begin{tabular}{|lllll|}
\hline $\begin{array}{l}\text { Tobacco/Smoking } \\
\text { Behaviors }\end{array}$ & $\begin{array}{l}\text { Current } \\
\text { infection \% }\end{array}$ & $\begin{array}{l}\text { Previous } \\
\text { infection \% }\end{array}$ & $\begin{array}{l}\text { Population prevalence (e.g., Current } \\
\text { or Previous Infection) } \%\end{array}$ & $\begin{array}{c}\text { P- } \\
\text { Values }\end{array}$ \\
\hline $\begin{array}{l}\text { Current cigarette } \\
\text { Use }\end{array}$ & $1.2 \%$ & $0.8 \%$ & $2.0 \%$ & 0.001 \\
Every day & $4.5 \%$ & $2.7 \%$ & $7.2 \%$ & \\
Some days & $4.9 \%$ & $1.8 \%$ & $6.6 \%$ & \\
Not at all & & & & 0.121 \\
$\begin{array}{l}\text { Current chewing } \\
\text { tobacco use }\end{array}$ & $6.1 \%$ & $0.8 \%$ & $7.0 \%$ & \\
Every day & $9.2 \%$ & $2.6 \%$ & $11.8 \%$ & \\
Some days & $4.5 \%$ & $1.7 \%$ & $6.2 \%$ & 0.329 \\
Not at all & & & & \\
$\begin{array}{l}\text { Current vaping or e- } \\
\text { cigarette use }\end{array}$ & $2.8 \%$ & $0 \%$ & $2.7 \%$ & \\
Every day & $3.2 \%$ & $3.2 \%$ & $6.4 \%$ & \\
Some days & $4.6 \%$ & $1.7 \%$ & $6.3 \%$ & \\
Not at all & & & & \\
\hline
\end{tabular}

In the bivariate logistic regression model (see Table 3), compared to individuals self-reporting excellent health status, those reporting good $(\mathrm{OR}=1.42,95 \% \mathrm{Cl}: 1.05-1.91)$ or fair $(\mathrm{OR}=1.78,95 \% \mathrm{Cl}$ : $1.23-2.57)$ health status had significantly higher positivity rates. Those who reported "poor" health status did not have higher positivity rates, however they represented only $1.1 \%$ of the sample. With respect to tobacco use, whereas smoking cigarettes (daily or on some days) was inversely associated with infection rates $(\mathrm{OR}=0.49,95 \% \mathrm{Cl}: 0.32-0.74)$; the use of chewing tobacco products was positively associated with infection (OR $=2.17,95 \% \mathrm{Cl}$ : $1.26-3.72)$. The use of vaping/e-cigarettes was not associated with infection rates $(\mathrm{OR}=0.654,95 \% \mathrm{Cl}: 0.32-1.35)$. 
Table 3

Binary logistic regression, controlling for age, sex, race, ethnicity, and randomized or non-randomized sample examining relationship between infection and self-reported health status and tobacco use.

\begin{tabular}{|llll|}
\hline & Odds Ratio & $\mathbf{9 5 \%}$ Confidence Intervals & P-Value \\
\hline Excellent Health Status & Reference & Reference & Reference \\
\hline Very Good Health Status & 1.02 & $0.75-1.39$ & 0.896 \\
\hline Good Health Status & 1.42 & $1.05-1.91$ & 0.022 \\
\hline Fair Health Status & 1.78 & $1.23-2.57$ & 0.002 \\
\hline Poor Health Status & 1.83 & $0.82-4.07$ & 0.137 \\
\hline Any Cigarette Smoking & 0.49 & $0.32-0.74$ & $<0.001$ \\
\hline Chewing Tobacco & 2.17 & $1.26-3.72$ & 0.005 \\
\hline Vaping/e-cigarettes & 0.654 & $0.32-1.35$ & 0.250 \\
\hline
\end{tabular}

\section{Conclusions}

As self-reported health status declined, the risk of being infected increased in a community-based population undergoing seroprevalence testing in nonclinical settings. The use of cigarette, especially daily, was inversely associated with infection. This finding is biologically plausible given the growing body of evidence that nicotine has an effect on the angiotensin-converting enzyme (ACE 2) (17). Though the literature is complex and somewhat inconsistent, nicotine may bind with the ACE 2 receptor, which is associated with a reduction in ACE 2 levels in multiple organs. Thus, nicotine may reduce the number of binding sites available to SARS-CoV-2, which also binds to the ACE 2 receptor (16).

However, previous research suggests that those who smoke and become infected are more likely to have severe disease and overall poor outcomes as documented in an early study in China, in which more smokers had more severe disease requiring hospitalization and mechanical ventilation as compared to those who did not smoke (18). In the same study, smokers were more likely to die as compared to nonsmokers (18). Our findings provide a new view of the relationship between smoking and SARS-CoV-2 infections but is consistent with the growing body of evidence that found similar inverse associations between smoking and hospitalization for COVID-19, which has been documented in other countries (18, 19). As with other studies, we lacked granular data on the duration and/or quantity of nicotine intake underpinning the observed associations. We do however know that co-morbid conditions associated with severe disease and death such as heart disease, diabetes, obesity, cerebrovascular disease, hypertension, COPD or other lung conditions, kidney disease are all associated with or worsened by smoking (20).

This is the first population-based study to assess risk factors behavioral risk factors for SARS-CoV2 infection. As previously noted, data on the cumulative quantity and duration of cigarette smoking is 
lacking so it is not possible to identify how much exposure is needed that lead to this inverse association. In addition, this study did not collect data on individuals that were institutionalized, generalizing to these excluded groups should be done with caution.

This study found an inverse epidemiological association between smoking and infection with SARS-CoV2 that is contrary to findings from predominantly severely ill COVID-19 patients. More study is needed to better understand the complex interaction between smoking, SARS-CoV-2 infection, and adverse clinical outcomes.

\section{Declarations}

The Institutional Review Board (IRB) at Indiana University deemed the study as exempt from human subjects research under the public health surveillance exemption. All experimental protocols were approved by Indiana University and the Indiana State Department of Health. Informed consent was obtained from all subjects or if subjects were under 18, from a parent and/or legal guardian.

\section{Consent for publication:}

Not applicable

The datasets used and/or analyzed during the current study are available from the corresponding author on reasonable request.

The authors declare that they have no competing interests.

\section{Funding:}

Indiana State Department of Health (contract \# 00043143)

\section{Acknowledgements:}

The Indiana Department of Health and all of the dedicated state and local health department staff who made this work possible.

\section{Author Contributions:}

(1)TD conceptualized the work, interpreted the data and wrote the main manuscript text. (2)WF acquired the data, interpreted the data, and prepared the tables. (3) KW interpreted the data, critically revised the manuscript. (4)BD interpreted the data, critically revised the manuscript. (5\&6) CY and PH interpreted the 
data and critically revised the manuscript. (7)NM conceptualized and designed the work, interpreted the data and critically revised the manuscript.

\section{References}

1. Kashyap, V. K., Dhasmana, A., Massey, A., Kotnala, S., Zafar, N., Jaggi, M., Yallapu, M. M., \& Chauhan, S. C. (2020). Smoking and COVID-19: Adding Fuel to the Flame. International journal of molecular sciences, 21(18), 6581. https://doi.org/10.3390/ijms21186581

2. Cucinotta, D., Vanelli, M. WHO Declares Covid-19 a Pandemic. Acta Biomed. 2020 Mar 19;19 (1) 157-160. Doi: 10.23750/abm.v91i1.9397.

3. Zhou F, Yu T, Du R, et al. Clinical course and risk factors for mortality of adult inpatients with COVID19 in Wuhan, China: a retrospective cohort study [published correction appears in Lancet. 2020 Mar 28;395(10229):1038] [published correction appears in Lancet. 2020 Mar 28;395(10229):1038]. Lancet. 2020;395(10229):1054-1062. doi:10.1016/S0140-6736(20)30566-3

4. Zhou Y, Zhang Z, Tian J, Xiong S. Risk factors associated with disease progression in a cohort of patients infected with the 2019 novel coronavirus. Ann Palliat Med. 2020;9(2):428-436. doi:10.21037/apm.2020.03.26

5. World Health Organization, Report of the WHO-China Joint Mission on Coronavirus Disease 2019 (COVID-19). 16-24 February 2020. Retrieved from https://www.who.int/docs/defaultsource/coronaviruse/who-china-joint-mission-on-covid-19-final-report.pdf

6. Wu, J.T., Leung, K., Bushman, M. et al. Estimating clinical severity of COVID-19 from the transmission dynamics in Wuhan, China. Nat Med 26, 506-510 (2020). https://doi.org/10.1038/s41591-020-08227

7. Centers for Disease Control and Prevention, Coronavirus Disease 2019 (COVID-19) Groups at Higher Risk for Severe Illness. Retrieved from: https://www.cdc.gov/coronavirus/2019-ncov/need-extraprecautions/groups-at-higher-risk.html on May 18, 2020

8. CDC COVID-19 Response Team. Preliminary Estimates of the Prevalence of Selected Underlying Health Conditions Among Patients with Coronavirus Disease 2019 - United States, February 12March 28, 2020. MMWR Morb Mortal Wkly Rep. 2020;69(13):382-386. Published 2020 Apr 3. doi:10.15585/mmwr.mm6913e2

9. Centers for Disease Control and Prevention, National Center for Chronic Disease Prevention and Health Promotion, Division of Population Health. Chronic Disease Indicators (CDI) Data [online]. 2016 [accessed May 18, 2020]. URL: https://nccd.cdc.gov/cdi. May 8, 2020

10. Fu H, Feng D, Tang S, et al. Prevalence of Tobacco Smoking and Determinants of Success in Quitting Smoking among Patients with Chronic Diseases: A Cross-Sectional Study in Rural Western China. Int J Environ Res Public Health. 2017;14(2):167. Published 2017 Feb 9. doi:10.3390/ijerph14020167

11. United Health Foundation: America's Health Rankings: Annual Report. Retrieved from https://www.americashealthrankings.org/explore/annual/measure/Overall/state/IN May 18, 2020 
12. Centers for Disease Control and Prevention, Coronavirus Disease 2019 (COVID-19). People with certain medical conditions. Retrieved from https://www.cdc.gov/coronavirus/2019-ncov/need-extraprecautions/people-with-medical-conditions.html\#smoking October 4, 2020

13. Chaumont $M$, van de Borne $P$, Bernard $A$, et al. Fourth generation e-cigarette vaping induces transient lung inflammation and gas exchange disturbances: results from two randomized clinical trials. Am J Physiol Lung Cell Mol Physiol. 2019;316(5):L705-L719. doi:10.1152/ajplung.00492.2018.

14. Indiana State Department of Health, Indiana's Tobacco Burden. Retrieved from: https://www.in.gov/isdh/tpc/files/Indiana\%27s\%20Tobacco\%20Burden_12_31_2019.pdf May 8, 2020.

15. Centers for Disease Control and Prevention, Behavioral Risk Factor Surveillance System. Retrieved from https://www.cdc.gov/brfss/questionnaires/pdf-ques/2019-BRFSS-Questionnaire-508.pdf November 30, 2020

16. Menachemi N, Yiannoutsos CT, Dixon BE, et al. Population Point Prevalence of SARS-CoV-2 Infection Based on a Statewide Random Sample - Indiana, April 25-29, 2020. MMWR Morb Mortal Wkly Rep 2020;69:960-964. DOI: http://dx.doi.org/10.15585/mmwr.mm6929e1

17. Hartmann-Boyce J, Lindson N. The Role of Nicotine in COVID-19 Infection. Oxford Centre for Evidence-Based Medicine, Nuffield Department of Primary Care Health Sciences, University of Oxford. Retrieved from: https://iu.zoom.us/j/96640902626 June 26, 2020

18. Ivan Berlin, MD, PhD, Daniel Thomas, MD, Anne-Laurence Le Faou, MD, PhD, Jacques Cornuz, MD, MPH, COVID-19 and Smoking, Nicotine \& Tobacco Research, Volume 22, Issue 9, September 2020, Pages 1650-1652, https://doi.org/10.1093/ntr/ntaa059

19. Robertson S. An inverse relationship between smoking and COVID-19. Retrieved from https://www.news-medical.net/news/20200615/An-inverse-relationship-between-smoking-andCOVID-19.aspx August 29, 2020.

20. Centers for Disease Control and Prevention: Coronavirus Disease 2019: Covid-19: People with Certain Medical Conditions. Retrieved from: https://www.cdc.gov/coronavirus/2019-ncov/need-extraprecautions/people-with-medical-conditions.html on September 2, 2020. 